\title{
Prescription rate of medications potentially contributing to lower urinary tract symptoms and detection of adverse reactions by prescription sequence symmetry analysis
}

\author{
Masako Hashimoto ${ }^{1,2+}$, Kanako Hashimoto $^{1 \dagger}$, Fumihiko Ando ${ }^{3}$, Yoshiaki Kimura ${ }^{1,5}$, Keisuke Nagase ${ }^{4}$ and Kunizo Arai $^{{ }^{*}}$
}

\begin{abstract}
Background: The lower urinary tract symptoms (LUTS) increases with age and can have a significant effect on the quality of life of the patients. Elderly patients, who are often characterized by a decline in physiological functional and polypharmacy, are susceptible to adverse drug reactions to pharmacotherapy. LUTS can also be a side effect of medication. The purpose of this study was to investigate the possible association between the initiation of LUTS-causing drug therapy and the onset of LUTS.
\end{abstract}

Methods: Drug dispensing data at the individual level were retrieved from the CISA (Platform for Clinical Information Statistical Analysis: http://www.cisa.jp) database. A retrospective study was conducted by reviewing patients with LUTS who were dispensed drugs that increased the risk of LUTS between April 2011 and March 2012. Prescription sequence symmetry analysis (PSSA) was employed to investigate the associations between the dispensing of medicines of LUTS and that of LUTS-causing drugs.

Results: LUTS-causing drugs were frequently dispensed to patients with LUTS. The use of medications potentially contributing to LUTS was associated with polypharmacy [number of prescription drugs:12.13 \pm 6.78 (user) vs. $5.67 \pm 5.24$ (nonuser)] but not patient age [ age: $(71.38 \pm 13.28$ (user) vs. $70.45 \pm 14.80$ (nonuser)]. Significant adverse drug events were observed the use of donepezil, cyclophosphamide, antiparkinson drugs, antidepressant, diazepam, antipsychotic drugs for peptic ulcer, tiotropium bromide, and opioids.

Conclusions: The use of prescription LUTS-causing drugs was correlated with polypharmacy. The adverse drug events associated with LUTS-causing drugs were highly prevalent in elderly patients. To prevent of adverse drug events in patients with LUTS, pharmacists and physicians should regularly review medication lists and reduce the prescribed medicines.

Keywords: Lower urinary tract symptoms, Voiding symptoms, Storage symptoms, Prescription sequence symmetry analysis, Adverse reactions

\section{Background}

The prevalence of lower urinary tract symptoms (LUTS) include voiding symptoms and storage symptoms. Voiding symptoms and storage symptoms are conditions in which the bladder is unable to store urine properly (storage symptoms) or empty properly (voiding symptoms) [1].

\footnotetext{
* Correspondence: arai@p.kanazawa-u.ac.jp

${ }^{\dagger}$ Equal contributors

${ }^{1}$ Faculty of Pharmacy, Institute of Medical, Pharmaceutical and Health Sciences, Kanazawa University, Kakuma-machi, Kanazawa 920-1192, Japan Full list of author information is available at the end of the article
}

Storage symptoms can show as stress incontinence, urge incontinence, mixed storage symptoms, or overflow incontinence. Voiding symptoms presents as a wide range of symptoms that can include difficulty in bladder emptying, urinary hesitancy, slow or weak urine stream urinary retention and dribbling of urine. In men, these symptoms can also be because of an enlarged prostate, a condition known as benign prostatic hypertrophy (BPH).

The LUTS increases with age and can have a significant effect on the quality of life [2]. Older individuals frequently 
have comorbid disorders and take medications that can impair continence via direct effects upon lower urinary tract function, alteration in volume status and urine excretion, cognitive impairment, and impairment of the functional ability to use a toilet. The hospitalized elderly with incontinence are more likely to have impaired mobility, dementia, and delirium and receive psychoactive medications than their continent patients [3].

Elderly patients, who are often characterized by a decline in physiological function and polypharmacy, are susceptible to adverse drug reactions to pharmacotherapy $[4,5]$. Poor compliance is most likely to occur among nonhospitalized patients who receive long-term prescriptions of preventive medications. In addition, $40 \%-50 \%$ of elderly patients have been unable to take medication as prescribed by their physicians $[6,7]$.

LUTS can also be a side effect of medication $[8,9]$. Recently, significant associations between the initiation of calcium channel blockers, angiotensin-converting enzyme inhibitors, angiotensin II receptor blockers, hormone replacement therapies, and hypnotic-sedatives and the subsequent initiation of oxybutynin, which is used to manage urgency incontinence, were reported [10]. Moreover, drugs that induce incontinence include alpha 1adrenoceptor antagonists, antipsychotics, benzodiazepines, antidepressants, and hormone replacement therapies in postmenopausal women [11-13].

Several studies illustrated that individuals with urinary incontinence are more likely to be using certain medicines, including antihistamines, beta agonists, angiotensinII receptor blockers, anticonvulsants, and benzodiazepines [14]. But a causal link between the use of these medicines and urinary incontinence has not been established.

We examined the prescription rate of drugs that can cause LUTS to patients who ultimately develop LUTS. In addition, we performed prescription sequence symmetry analysis (PSSA) to investigate the risk of LUTS caused by drug side effects.

\section{Methods}

\section{Study design and data source}

Drug dispensing data at the individual level were retrieved from the CISA (Platform for Clinical Information Statistical Analysis: http://www.cisa.jp) database, which contains substantial clinical information obtained in Japan. Currently, data from CISA are provided in a fully anonymized form to 14 national university hospitals. In the CISA database, each prescription record contains basic patient characteristics (anonymous identifier, gender, and date of birth) and information on the drug name, anatomical therapeutic chemical code, dosage, and dispensing date.

This study identified patients with LUTS who were coded according to the 10th revision of the International Statistical Classification of Diseases and Related Health
Problems. Medicines known to cause LUTS were identified from drug information resources. The Medicines Handbook [15] was searched to identify medicines with the adverse drug reaction "LUTS." Finally, published articles were identified and reviewed to identify any additional medicines associated with LUTS. The list of medicines associated with LUTS that were included in the study is presented in Table 1.

Medications for LUTS were classified three groups, drugs for the management of storage symptoms, drugs for the management of voiding symptoms, and drugs for the management of mixed urinary dysfunction, as shown in Additional file 1.

A retrospective study was conducted by reviewing patients with LUTS who were dispensed drugs associated with LUTS between April 2011 and March 2012.

The data were stratified to determine the number of drugs and/or the potential LUTS risk of such drugs used by patients with LUTS. To determine whether associations could be detected between the number of medications and age against the use of LUTS-causing medication, Student's $t$-test was performed to compare the use and nonuse of targeted medications potentially causing or exacerbating LUTS.

\section{PSSA}

PSSA, as developed by Hallas, is an effective surveillance tool for drug-associated adverse reactions [16,17]. PSSA has also been employed in previous studies investigating associations between the uses of certain targeted drugs. The validity of PSSA was confirmed by previous research [18-22].

In PSSA, the date of the first prescription of an LUTScausing drug (index drug) and that of the first medication used to manage LUTS (marker drug) are determined for each patients. A 6-month "waiting time" was imposed as a baseline period to ensure that the marker drug was indeed newly prescribed. The ratio of the number of patients with initiated LUTS-causing drug therapy before the initiation of LUTS treatment was compared with the number of patients who initiated LUTS-causing drug therapy after the start of LUTS treatment.

\section{Statistical analysis for PSSA}

The ratio of patients who initiated marker drug treatment after the initiation of index drug treatment (index $\rightarrow$ marker) to those who initiated marker drug treatment before the initiation of index drug treatment (marker $\rightarrow$ index) was defined as the crude sequence ratio (SR). PSSA could be sensitive to prescribing trends, such as rapid increases in marker drug use, over time. To adjust for such temporal trends, a null-effect SR (SRnull) was calculated. SRnull is the expected SR in the absence of a causal association, given the incident medicine use 
Table 1 Number of patients with LUTS prescribed medicines that can cause LUTS

\begin{tabular}{llll}
\hline Medication class & Medicine & \multicolumn{2}{l}{$\begin{array}{l}\text { Prevalence } \\
\mathbf{n}(\%)\end{array}$} \\
\hline Anti-dementia drugs & Donepezil & 272 & 14.1 \\
Intestinal Lavage Solution & Intestinal Lavage Solution & 44 & 2.3 \\
Decongestants & Tranilast & 18 & 1.7 \\
and antiallergics & Ketotifen & 15 & \\
& Other & $※$ & \\
Antineoplastic agents & Cyclophosphamide & 17 & 1.6
\end{tabular}

(b) Medicines that caused the voiding symptoms

\begin{tabular}{llll}
\hline Medication class & Medicine & \multicolumn{2}{l}{$\begin{array}{l}\text { Prevalence } \\
\mathbf{n}(\%)\end{array}$} \\
\hline Anti-parkinson drugs & Trihexyphenidyl & 32 & 13.7 \\
& Biperiden & 56 & \\
& Amantadine & 93 & \\
& Levodopa/Benserazide & 72 & \\
& Other & $※$ & \\
Antidepressants & Mianserin & 58 & 13.8 \\
& Paroxetine e & 119 & \\
& Fluvoxamine & 61 & \\
& Milnacipran & 29 &
\end{tabular}

Anxiolytics

Diazepam

other

Antipsychotics

$\begin{array}{lcc}\text { Chlorpromazine } & 30 & 13.3 \\ \text { Risperidone } & 108 & \\ \text { Levomepromazine } & 36 & \\ \text { Sulpiride } & 68 & \\ \text { other } & ※ & \\ \text { Cimetidine } & 34 & 5.6 \\ \text { Scopolamine } & 67 & \\ \text { butylbromide } & & \\ \text { other } & ※ & \\ \text { Tiotropium bromide } & 129 & 6.7 \\ \text { other } & ※ & \\ \text { Diphenhydramine } & 17 & 3.3 \\ \text { Chlorpheniramine } & 40 & \\ \text { other } & ※ & \\ \text { Cibenzoline } & 27 & 2.0 \\ \text { other } & ※ & \\ \text { Amezinium metilsulfate } & 20 & 4.8 \\ \text { Droxidopa } & 48 & \\ \text { Midodrine } & 24 & \\ \text { other } & ※ & \end{array}$

Drugs for peptic ulcer and gastro-oesophageal reflux disease (GORD)

Other drugs for obstructive airway diseases, inhalants

Antihistamines for systemic use

Antiarrhythmics, class I and III

Cardiac stimulants excl. cardiac glycosides
Table 1 Number of patients with LUTS prescribed medicines that can cause LUTS (Continued)

\begin{tabular}{llll}
\hline Opioids & Morphine & 19 & 12.4 \\
& Codeine phosphate & 39 & \\
& Oxycodone & 172 & \\
& Other & $※$ & \\
&
\end{tabular}

and events in the background population. SRnull is the expected SR of an incidence trend when there is no causal relationship between the index and marker drugs, providing a background rate for the chronological sequence of two drugs. In this study, we computed the probability of an index drug to marker drug sequence for each user of an LUTS drug at the first prescription. The overall probability of a medicine for treating LUTS, $\mathrm{Pa}$, was generated by weighting the number of incident users on each prescribing date of drugs for LUTS and averaging the value over all days. SRnull was then computed as $\mathrm{Pal}$ $(1-P a)$. An adjusted SR was obtained by dividing the crude SR by SRnull, and 95\% confidence intervals (CIs) were determined with a normal approximation to the binomial distribution. All analyses were performed using Ekuseru-Toukei 2012 (Social Survey Research Information Co., Ltd. Tokyo, Japan).

\section{Ethics statement}

The study was approved by the Institutional Review Board of CISA (receipt number: HA1405004).

\section{Results}

Characteristics of the participants (age, sex, number of drug prescriptions)

Table 2 shows the characteristics of the participants and distribution of the types of LUTS. The mean age of the patients was $70.52 \pm 14.60$ years, and patients consumed an average of $6.16 \pm 5.38$ different medications. The proportion of male patients with voiding symptoms was large $(91.5 \%)$. In men, these symptoms can also be because of $\mathrm{BPH}$.

\section{Percentage of prescription agents that were responsible} for urinary disturbance and the efficacy classification The proportion of patients who were using medications potentially contributing to urinary symptoms was $7.7 \%$, with a mean of one incriminating drug per patient (Table 3). The top five medication classes were donepezil (14.1\% in user of LUTS-causing drugs), antiparkinson drugs (13.7\%), antidepressants (13.8\%), antipsychotics (13.3\%), and opiates/narcotics (12.4\%) (Table 1). 
Table 2 Patient characteristics and frequency of use of medications potentially contributing to urinary symptoms

\begin{tabular}{lllll}
\hline & All & Storage symptoms & Voiding symptoms & Mixed urinary symptoms \\
\hline Number of patients (\%) & $17,824(100 \%)$ & $5,127(28.8 \%)$ & $10,967(61.5 \%)$ & $1730(9.7 \%)$ \\
Male (\%) & $13,777(77.2 \%)$ & $2,159(42.1 \%)$ & $10,031(91.5 \%)$ & $1,587(91.7 \%)$ \\
Age (Mean \pm SD) & $70.52 \pm 14.60$ & $64.90 \pm 20.00$ & $72.56 \pm 10.98$ & $74.22 \pm 10.60$ \\
Number of prescription drugs (Mean \pm SD) & $6.16 \pm 5.38$ & $6.04 \pm 5.58$ & $6.01 \pm 5.26$ & $7.50 \pm 5.27$ \\
\hline
\end{tabular}

The relationships of patient age and polypharmacy with the use of medications potentially contributing to urinary symptoms

The use of medications potentially contributing to LUTS was associated with polypharmacy $(12.13 \pm 6.78$ vs. $5.67 \pm$ $5.24, \mathrm{P}$ value $<0.0001)$ but not patient age $(71.38 \pm 13.28$ vs. $70.45 \pm 14.8, \mathrm{P}$ value $=0.0244)$. Among patients with voiding symptoms, those who used LUTS-causing drugs were younger than nonusers $(69.79 \pm 13.58$ vs. $72.81 \pm$ 10.71 , P value $<0.0001)$. An opposing trend was observed among patients with storage symptoms (77.35 \pm 10.88 vs. $64.30 \pm 20.34$, P value <0.0001) (Table 3)

\section{Likelihood of incident medicine after initiation of a medicine that may be associated with LUTS}

PSSA identified significant associations between the initiation of opioids [oxycodone (adjusted SR: 1.20; 95\% CI: 1.03-1.41), morphine (adjusted SR: 1.29; 95\% CI: 1.141.45)], donepezil (adjusted SR: 1.98; 95\% CI: 1.57-2.50), intestinal lavage solution (adjusted SR: 1.86; 95\% CI: 1.65-2.10), cyclophosphamide (adjusted SR: 1.52; 95\% CI: 1.14-2.04), levodopa/benserazide (adjusted SR: 1.82; 95\% CI: 1.18-2.81), selective serotonin reuptake inhibitor (SSRI) [paroxetine (adjusted SR: 1.77; 95\% CI: 1.33-2.36)], serotonin and norepinephrine reuptake inhibitor (SNRI) [milnacipran (adjusted SR: 2.10; 95\% CI: 1.28-3.45)], diazepam (adjusted SR: 1.73; 95\% CI: 1.46-2.06), serotonin dopamine antagonist [risperidone (adjusted SR: 1.55; 95\% CI: 1.34-1.79)], levomepromazine (adjusted SR: 2.20; 95\% CI: 1.34-1.79), sulpiride (adjusted SR: 1.32; 95\% CI: 1.01-1.72), histamine H2-receptor antagonists [cimetidine (adjusted SR: 1.99; 95\% CI: 1.24-3.20)], scopolamine butylbromide (adjusted SR: 1.72; 95\% CI 1.55-1.92), anticholinesterases (tiotropium bromide (adjusted SR: 1.75; 95\% CI: 1.42-2.16), cibenzoline (adjusted SR: 2.97; 95\% CI: 1.92-4.59), and amezinium metilsufate (adjusted SR: 1.89; 95\% CI: 1.10-3.26) and the subsequent initiation of medication for managing LUTS (Table 4). When the time window among the events was restricted to 3 months, the results were largely consistent with the primary analyses.

\section{Discussion}

The number of prescription drugs, but not age, was significantly different between users $(12.13 \pm 6.70)$ and nonusers $(5.67 \pm 5.24)$ of medicines that can cause urinary symptoms.
Polypharmacy, inappropriate prescribing, and adverse drug events are highly prevalent in elderly patients [23]. The characteristics of patients with LUTS included old age (average age, $70.52 \pm 14.60$ ) and male sex. Voiding symptoms was noted in $57.9 \%$ of women, presumably because of declines in muscle strength, such as pelvic floor muscle strength, with age. In men, the prevalence of high outlet obstruction (91.5\%) predominated as a result of prostate enlargement with age.

The typical elderly patient with LUTS typically receives medication for other comorbidities. Elderly patients taking five to eight drugs were reported to be at greater risk of adverse drug reaction related hospitalization than those taking zero to four drugs [23]. However, a meaningful correlation was not found between age and the number of the prescription drugs used by patients with LUTS (data not shown) in this study. This result may be responsible for the findings that patients with LUTS were old (average age, $70.52 \pm 14.60$ ) and presented with several comorbid conditions requiring medication (average number of prescription drugs, 6.16 \pm 5.38 ).

In total, $7.7 \%$ of patients were prescribed medications potentially contributing to urinary symptoms. Donepezil (14.1\%), antiparkinson drugs (13.7\%), antidepressants (13.8\%), and antipsychotics (13.3\%) were frequently prescribed, reflecting the rate of complications in older people. Patients who used medicines that can cause storage symptoms, such as donepezil, were older than nonusers. In contrast, patients who used medicines that can cause voiding symptoms, such as antiparkinson drugs (13.7\%), antidepressants (13.8\%), and antipsychotics, were younger than their nonusing counterparts. The prevalence of incontinence in the elderly with dementia is higher than that of persons without dementia. Multiple comorbid diseases, such as depression, Parkinson's disease, psychotic disorders, and medications are possible risk factors for voiding symptoms.

In this study, we demonstrated that the side effects of donepezil, cyclophosphamide, amantadine, levodopa/benserazide, paroxetine, fluvoxamine,milnacipran, diazepam, risperidone,levomepromazine,sulpiride, cimetidine, scopplamine butylbromide, tiotropium bromide, and opioids were associated with LUTS. Risperidone therapy has been associated with storage symptoms (28\% in some cases) [24]. Risperidone primarily acts as an antagonist of serotonin type 2A (5HT2a) and D2-dopamine receptors, and 
Table 3 Relationship between age and number of medications used for patients prescribed medications potentially contributing to urinary symptoms

\begin{tabular}{|c|c|c|c|c|c|c|c|c|c|c|c|c|}
\hline & \multicolumn{3}{|l|}{ All } & \multicolumn{3}{|c|}{ Storage symptoms } & \multicolumn{3}{|c|}{ Voiding symptoms } & \multicolumn{3}{|c|}{ Mixed urinary symptoms } \\
\hline & user & nonuser & $P$ value & user & nonuser & $P$ value & user & nonuser & $P$ value & user & nonuser & $P$ value \\
\hline Number of patients (\%) & $1,365(7.7 \%)$ & 16,459 & - & $234(4.6 \%)$ & 4,893 & - & $907(8.3 \%)$ & 10,060 & - & $224(12.9 \%)$ & 1,506 & - \\
\hline Age (Mean $\pm S D)$ & $71.38 \pm 13.28$ & $70.45 \pm 14.80$ & 0.0244 & $77.35 \pm 10.88$ & $64.30 \pm 20.34$ & $<0.0001$ & $69.79 \pm 13.58$ & $72.81 \pm 10.71$ & $<0.0001$ & $71.63 \pm 12.56$ & $74.60 \pm 10.27$ & 0.0002 \\
\hline $\begin{array}{l}\text { Number of prescription } \\
\text { drugs }(\text { Mean } \pm \text { SD) }\end{array}$ & $12.13 \pm 6.78$ & $5.67 \pm 5.24$ & $<0.0001$ & $9.95 \pm 6.55$ & $5.86 \pm 5.53$ & $<0.0001$ & $12.47 \pm 6.82$ & $5.43 \pm 5.10$ & $<0.0001$ & $13.05 \pm 6.43$ & $6.67 \pm 5.08$ & $<0.0001$ \\
\hline
\end{tabular}


Table 4 Likelihood of incident medicine after initiation of a medicine that may be associated with LUTS (April 2006-January 2014)

\begin{tabular}{|c|c|c|c|c|c|c|c|c|}
\hline & \multirow[t]{2}{*}{ Medicine } & \multirow{2}{*}{$\begin{array}{l}\text { Users of } \\
\text { medicine }\end{array}$} & \multicolumn{3}{|c|}{ In the 12 months sequence } & \multicolumn{3}{|c|}{ In the 3 months sequence } \\
\hline & & & $\begin{array}{l}\text { Index } \rightarrow \text { Marker/ } \\
\text { Marker } \rightarrow \text { Index (n) }\end{array}$ & Adjusted SR & $95 \% \mathrm{Cl}$ & $\begin{array}{l}\text { Index } \rightarrow \text { Marker/ } \\
\text { Marker } \rightarrow \text { Index (n) }\end{array}$ & Adjusted SR & $95 \% \mathrm{Cl}$ \\
\hline Anti-dementia drugs & Donepezil & 7,825 & 210/109 & $1.98^{*}$ & $1.57-2.50$ & $175 / 68$ & 1.32 & $1.00-3.50$ \\
\hline Intestinal Lavage Solution & Intestinal Lavage Solution & 54,014 & $722 / 404$ & $1.86^{*}$ & $1.65-2.10$ & $528 / 228$ & 1.17 & $1.06-2.82$ \\
\hline \multirow[t]{2}{*}{ Decongestants and antiallergics } & Tranilast & 7,161 & $0 / 3$ & NA & NA & & NA & NA \\
\hline & Ketotifen & 4,644 & $0 / 0$ & NA & NA & & NA & NA \\
\hline Antineoplastic agents & Cyclophosphamide & 7,787 & $111 / 77$ & $1.52^{*}$ & $1.14-2.04$ & $64 / 62$ & 1.42 & $0.768-1.55$ \\
\hline \multirow[t]{4}{*}{ Antiparkinson } & Trihexyphenidyl & 1,844 & $24 / 21$ & 1.14 & $0.63-2.04$ & $21 / 13$ & 2 & $0.804-3.21$ \\
\hline & Biperiden & 3,177 & $59 / 41$ & 1.39 & $0.93-2.07$ & $62 / 29$ & 1.55 & $1.33-3.12$ \\
\hline & Amantadine & 2,155 & $99 / 65$ & $1.53^{*}$ & $1.12-2.09$ & $86 / 47$ & 1.42 & $1.29-2.62$ \\
\hline & Levodopa/Benserazide & 1,652 & $58 / 32$ & $1.82^{*}$ & $1.18-2.81$ & $49 / 15$ & 1.78 & $1.84-5.86$ \\
\hline \multirow[t]{4}{*}{ Antidepressants } & Mianserin & 1,999 & $73 / 79$ & 0.94 & $0.68-1.29$ & $71 / 65$ & 1.4 & $0.794-1.56$ \\
\hline & Paroxetine & 9,021 & $139 / 70$ & $1.77^{*}$ & $1.33-2.36$ & $120 / 57$ & 1.37 & $1.37-2.57$ \\
\hline & Fluvoxamine & 5,178 & $65 / 39$ & 1.48 & $0.99-2.21$ & $62 / 28$ & 1.56 & $1.26-3.08$ \\
\hline & Milnacipran & 2,578 & $52 / 22$ & $2.10^{*}$ & $1.28-3.45$ & $49 / 13$ & 1.84 & $1.82-6.18$ \\
\hline Anxiolytic & Diazepam & 57,776 & $658 / 450$ & $1.44^{*}$ & $1.28-1.63$ & $602 / 336$ & 1.14 & $1.55-2.02$ \\
\hline \multirow[t]{4}{*}{ Antipsychotic } & Chlorpromazine & 4,448 & $121 / 117$ & 1.09 & $0.85-1.41$ & $114 / 91$ & 1.32 & $1.00-1.74$ \\
\hline & Risperidone & 14,330 & $466 / 310$ & $1.55^{*}$ & $1.34-1.79$ & $491 / 276$ & 1.16 & $1.58-2.12$ \\
\hline & Levomepromazine & 2,391 & $59 / 26$ & $2.20^{*}$ & $1.34-1.79$ & $45 / 16$ & 1.77 & $1.54-4.82$ \\
\hline & Sulpiride & 7,600 & $126 / 91$ & $1.32^{*}$ & $1.01-1.72$ & $112 / 65$ & 1.36 & $1.21-2.22$ \\
\hline \multirow[t]{2}{*}{ Drugs for peptic ulcer } & Cimetidine & 2,886 & $53 / 25$ & $1.99^{*}$ & $1.24-3.20$ & $48 / 18$ & 1.72 & $1.46-4.30$ \\
\hline & Scopolamine butylbromide & 97,755 & $909 / 523$ & $1.72^{*}$ & $1.55-1.92$ & $703 / 391$ & 1.13 & $1.57-2.02$ \\
\hline Anticholinergic bronchodilator & Tiotropium bromide & 5,179 & 234/135 & $1.75^{*}$ & $1.42-2.16$ & $213 / 94$ & 1.27 & $1.80-2.92$ \\
\hline \multirow[t]{2}{*}{ Antihistaminic } & Chlorpheniramine & 3,934 & $6 / 7$ & 0.87 & $0.29-2.60$ & & 3.72 & $0.342-4.74$ \\
\hline & Diphenhydramine & 13,073 & $0 / 2$ & NA & NA & $4 / 5$ & NA & NA \\
\hline Antiarrhythmics & Cibenzoline & 2,038 & $81 / 27$ & $2.97^{*}$ & $1.92-4.59$ & $73 / 21$ & 1.62 & $2.12-5.59$ \\
\hline \multirow[t]{3}{*}{ Cardiac stimulants excl. cardiac glycosides } & Amezinium metilsulfate & 1,665 & $38 / 20$ & $1.89^{*}$ & $1.10-3.26$ & $34 / 13$ & 1.89 & $1.38-4.94$ \\
\hline & Droxidopa & 650 & $15 / 17$ & 0.90 & $0.45-1.79$ & $13 / 11$ & 2.23 & $0.537-2.68$ \\
\hline & Midodrine & 1,346 & $24 / 20$ & 1.21 & $0.67-2.20$ & $23 / 12$ & 2.01 & $0.964-3.89$ \\
\hline
\end{tabular}


Table 4 Likelihood of incident medicine after initiation of a medicine that may be associated with LUTS (April 2006-January 2014) (Continued)

\begin{tabular}{lllllllll}
\hline Opioids & Morphine & 20,378 & $599 / 449$ & $1.29^{*}$ & $1.14-1.45$ & $564 / 370$ & 1.14 & $1.29-1.68$ \\
& Codeine phosphate & 11,223 & $234 / 223$ & 1.04 & $0.86-1.25$ & $192 / 137$ & $1.11-1.72$ \\
& Oxycodone & 6,370 & $334 / 289$ & $1.20^{*}$ & $1.03-1.41$ & $333 / 176$ & 1.2 & $1.64-2.37$
\end{tabular}

NA: Not applicable. *Statistical significant at an alpha level of 0.05 .

Index $\rightarrow$ Marker (causal): patients initiated marker drug therapy after initiating index drug treatment.

Marker $\rightarrow$ Index (noncausal): patients initiated index drug therapy after initiating marker drug treatment.

Index drug: Medicine associated with a risk of LUTS. Marker drug: Medication for treating LUTS.

Index drug: Medicine associated with a risk of LUTS. Marker drug: Medication for treating LUTS.
Number of participants who initiated a medicine associated with LUTS between 2005 and 2013.

Sequences of the incident index and marker drugs within 3 or 12 months.

Adjusted for the null-effect SR. 
it has a strong blockade effect on $\alpha-1$ and $\alpha-2$ adrenergic receptors. It may be that the antagonizing effect of risperidone on the $\alpha-1$ receptors of the internal bladder sphincter causes urinary retention [25]. Levomepromazine also may affect the $\alpha-1$ receptors of the internal bladder sphincter.

Sulpiride, a D2-selective dopaminergic receptor antagonist, increased bladder capacity in rats [26]. Dopaminergic systems have also been implicated in the control of the micturition reflex. D1-like dopaminergic receptors mediate forebrain-inhibitory effects on the micturition reflex, whereas D2-like dopaminergic receptors appear to be involved in excitatory regulation of the micturition reflex at the level of the brainstem. The prevalence of LUTS in patients with Parkinson disease (PD) is reported to be $27 \%-39 \%$ [27]. Levodopa is well established as the most effective drug for the symptomatic treatment of idiopathic or Lewy body PD. Amantadine is known to increase dopamine release, inhibit dopamine reuptake, and stimulate dopamine receptors, and it may possibly exert central anticholinergic effects [27].

Anticholinergic agents such as biperiden and trihexyphenidyl did not display meaningful peripheral antimuscarinic side effects. In contrast, the dopamine-related drugs levodopa and amantadine exerted side effects, suggesting that LUTS caused by such drugs is dependent on dopaminergic activity.

Milnacipran, a dual-action antidepressant that acts as a serotonin and norepinephrine reuptake inhibitor (SNRI), and paroxetine, a selective serotonin reuptake inhibitor (SSRI), have been linked to voiding symptoms. Animal studies suggested that incontinence secondary to serotonergic antidepressants could be mediated by $5 \mathrm{HT} 4$ receptors found on the bladder [28,29]. These drugs may affect serotonin uptake by $5 \mathrm{HT}$ receptors found on the bladder. Recently, experiments in cats illustrated that SNRIs suppress parasympathetic activity and increase sympathetic and somatic neural activity in the lower urinary tract [30]. The inhibition of serotonin and norepinephrine reuptake during bladder storage is believed to increase pudendal nerve output, resulting in increased tone of the rhabdosphincter and subsequently improved urethral closure. The SNRI duloxetine can significantly improve the quality of life of patients with stress urinary incontinence. In this study, voiding symptoms was caused by milnacipran, suggesting that milnacipran-associated voiding symptoms may have beneficial effects on urinary incontinence.

The most common medication class potentially contributing to LUTS is benzodiazepines (17.4\%) [31]. The mechanism by which other medicines cause incontinence is the activation of $N$-methyl- $D$-aspartate receptors in pontine micturition center (PMC) that are involved in the facilitation of voiding [32].
Men with LUTS or BPH who are treated with inhaled anticholinergic agents may develop acute urinary retention, but this cannot be quantified on the basis of the limited information available. Inhaled anticholinergic agents should be used when indicated in men with LUTS or BPH but close monitoring and patient education should be implemented [33]. Inhaled anticholinergic medication use in older men with chronic obstructive pulmonary disorder is associated with an increased risk of acute urinary retention [34].

The prevalence of opioid-induced dysuria in patients with advanced cancer-associated pain was $14.9 \%$ [35]. Urinary retention induced by systematically injected morphine was considered to result from the inhibition of bladder function mediated via $\mu$-opioid receptors of the micturition centers in the supraspinal and spinal regions [36].

Dementia and storage symptoms are common, and often coexisting, problems in older people. Anticholinergic drugs are used to treat urinary instability [37-39]. In this study, storage symptoms is expected to occur in patients treated with donepezil. The potential for worsened urinary continence is an important consideration when starting cholinesterase treatment in Alzheimer disease (AD).

Cyclophosphamide is metabolized to several moieties, including acrolein, which collects in the urinary bladder, leading to urothelial damage [40]. Cyclophosphamide causes cellular damage in bladder tissue that triggers an inflammatory response. Therefore, cyclophosphamide is commonly used in noninvasive rodent models of acute bladder pain [41].

The current study has highlighted the potential for the initiation of commonly used medicines to be associated with the subsequent initiation of drug treatment for LUTS and has provided an estimate of the risk of LUTS associated with these medicines. Prescribers should be aware of LUTS that occurs shortly after the initiation of new medicines, and the potential for an adverse event should be considered. For this reason, before the treatment for LUTS is started, the possible side effects of medications currently used for the patient should be reviewed and the planned treatment for LUTS should be adjusted if necessary.

\section{Conclusions}

We found that medicines that can cause urinary symptom were prescribed to patients taking many drugs and were associated with an increased risk of LUTS. The findings illustrated that polypharmacy and adverse drug events associated with LUTS-causing drugs were highly prevalent in patients with LUTS. In addition, our study provides a basis for future investigations of whether milnacipran-associated voiding symptoms may be useful for the treatment of storage symptoms. 


\section{Additional file}

\section{Additional file 1: Medication for LUTS.}

\section{Competing interests}

The authors declare that they have no competing interests.

\section{Authors' contributions}

$\mathrm{MH}, \mathrm{KH}, \mathrm{KA}$ : study design, data analysis, drafting the manuscript. FA, KN, YK: study design, critical review of manuscript. All authors read and approved the final manuscript.

\section{Acknowledgements}

We thank Professor Takada M (Kinki University) for his significant help in the design of the study.

\section{Author details \\ ${ }^{1}$ Faculty of Pharmacy, Institute of Medical, Pharmaceutical and Health Sciences, Kanazawa University, Kakuma-machi, Kanazawa 920-1192, Japan. \\ ${ }^{2}$ Temari Pharmacy, 2-50 Kobu-machi, Kanazawa 920-0362, Japan. \\ ${ }^{3}$ Department of Medical Informatics, Kyoto University Hospital, 54 Kawaharacho, SyogoinSakyu-ku, Kyoto 606-8507, Japan. ${ }^{4}$ Department of Medical Informatic, Kanazawa University Hospital, 2-251 Takara-machi, Kanazawa 920-8641, Japan. ${ }^{5}$ Suisen Pharmacy, Fukui Pharmaceutical Association, 906 Matsuokagokuryo Yoshida-gun Eiheijicho-cho, Fukui 910-1193, Japan.}

Received: 5 July 2014 Accepted: 29 October 2014

Published online: 15 February 2015

\section{References}

1. Thom M, Campigotto M, Vemulakonda V, Coplen D, Austin PF: Management of lower urinary tract dysfunction: a stepwise approach. J Pediatr Urol 2012, 8:20-24.

2. Dubeau CE: The aging lower urinary tract. J Urol 2006, 175(3 Pt 2):S11-S15.

3. Offermans MP, Du Moulin MF, Hamers JP, Dassen T, Halfens RJ: Prevalence of urinary incontinence and associated risk factors in nursing home residents: a systematic review. Neurourol Urodyn 2009, 28:288-294.

4. Talasz H, Lechleitner M: Polypharmacy and incontinence. Z Gerontol Geriatr 2012, 45:464-467.

5. Spagnoli A, Ostino G, Borga AD, D'Ambrosio R, Maggiorotti P, Todisco E, Prattichizzo W, Pia L, Comelli M: Drug compliance andunreported drugs in the elderly. J Am Geriatr Soc 1989, 37:619-624.

6. Elsawy B, Higgins KE: The geriatric assessment. Am Fam Physician 2011, 83:48-56.

7. Shah BM, Hajjar ER: Polypharmacy, adverse drug reactions, and geriatric syndromes. Clin Geriatr Med 2012, 28:173-186.

8. Gnjidic D, Johnell K: Clinical implications from drug-drug and drug-disease interactions in older people. Clin Exp Pharmacol Physiol 2013, 40:320-325.

9. Gnjidic D, Hilmer SN, Blyth FM, Naganathan V, Waite L, Seibel MJ, McLachlan AJ, Cumming RG, Handelsman DJ, Le Couteur DG: Polypharmacy cutoff and outcomes: five or more medicines were used to identify community-dwelling older men at risk of different adverse outcomes. J Clin Epidemiol 2012, 65:989-995.

10. Kalisch Ellett LM, Pratt NL, Barratt JD, Rowett D, Roughead EE: Risk of medication-associated initiation of oxybutynin in elderly men and women. J Am Geriatr Soc 2014, 62:690-695.

11. Wuerstle MC, Van Den Eeden SK, Poon KT, Quinn VP, Hollingsworth JM, Loo RK, Jacobsen SJ: Contribution of common medications to lower urinary tract symptoms in men. Arch Intern Med 2011, 171:1680-1682.

12. Buser N, Ivic S, Kessler TM, Kessels AG, Bachmann LM: Efficacy and adverse events of antimuscarinics for treating overactive bladder: network meta-analyses. Eur Urol 2012, 62:1040-1060.

13. Meng E, Chang HY, Chang SY, Sun GH, Yu DS, Cha TL: Involvement of purinergic neurotransmission in ketamine induced bladder dysfunction. J Urol 2011, 186:1134-1141.

14. Afonso AS, Verhamme KM, Stricker BH, Sturkenboom MC, Brusselle GG: Inhaled anticholinergic drugs and risk of acute urinary retention. $B J U$ Int 2011, 107:1265-1272.

15. Takaku F, Yazaki Y: Chiryouyaku manual 2012. Tokyo: Igaku-shoin; 2012.

16. Hallas J: Evidence of depression provoked by cardiovascular medication: a prescription sequencesymmetry analysis. J Epidemiology 1996, 7:478-484.
17. Maclure M, Fireman B, Nelson JC, Hua W, Shoaibi A, Paredes A, Madigan D: When should case-only designs be used for safety monitoring of medical products? Pharmacoepidemiol Drug Saf 2012, 21(Suppl):150-161.

18. Takada M, Fujimoto M, Hosomi K: Difference in risk of gastrointestinal complications between users of enteric-coated and buffered low-dose aspirin. Int J Clin Pharmacol Ther 2014, 52:181-191.

19. Sehgal V, Bajwa SJ, Sehgal R, Bajaj A, Khaira U, Kresse V: Polypharmacy and potentially inappropriate medication use as the precipitating factor in readmissions to the hospital. J Family Med Prim Care 2013, 2:194-199.

20. Wahab IA, Pratt NL, Wiese MD, Kalisch LM, Roughead EE: The validity of sequence symmetry analysis (SSA) for adverse drug reaction signal detection. Pharmacoepidemiol Drug Saf 2013, 22:496-502.

21. Lai EC, Yang YH, Lin SJ, Hsieh CY: Use of antiepileptic drugs and risk of hypothyroidism. Pharmacoepidemiol Drug Saf 2013, 22:1071-1079.

22. Lai EC, Hsieh CY, Kao Yang YH, Lin SJ: Detecting potential adverse reactions of sulpiride in schizophrenic patients by prescription sequence symmetry analysis. PLoS One 2014, 9:e89795.

23. Marcum ZA, Amuan ME, Hanlon JT, Aspinall SL, Handler SM, Ruby CM, Pugh MJ: Prevalence of unplanned hospitalizations caused by adverse drug reactions in older veterans. J Am Geriatr Soc 2012, 60:34-41.

24. Agarwal V: Urinary incontinence with risperidone. J Clin Psychiatry 2000, 61:219.

25. Hergüner S, Mukaddes NM: Risperidone-induced double incontinence. Prog Neuropsychopharmacol Biol Psychiatry 2008, 32:1085-1086.

26. Yokoyama O, Yoshiyama M, Namiki M, de Groat WC: Role of the forebrain in bladder overactivity following cerebral infarction in the rat. Exp Neurol 2000, 163:469-476.

27. Yeo $L$, Singh R, Gundeti M, Barua JM, Masood J: Urinary tract dysfunction in Parkinson's disease: a review. Int Urol Nephrol 2012, 44:415-424.

28. Votolato NA, Stern S, Caputo RM: Serotonergic antidepressants and urinary incontinence. Int Urogynecol J Pelvic Floor Dysfunct 2000, 11:386-388.

29. Aggarwal A, Khandelwal A, Jiloha RC: Milnacipran-associated urinary retention: a case report. J Clin Psychopharmacol 2010, 30:641.

30. Mariappan P, Ballantyne Z, N'Dow JM, Alhasso AA: Serotonin and noradrenaline reuptake inhibitors (SNRI) for stress urinary incontinence in adults. Cochrane Database Syst Rev 2005, 20:CD004742.

31. Kashyap M, Tu LM, Tannenbaum C: Prevalence of commonly prescribed medications potentially contributing to urinary symptoms in a cohort of older patients seeking care for incontinence. BMC Geriatr 2013, 13:57.

32. Kontani $\mathrm{H}$, Ueda $\mathrm{Y}$ : A method for producing overactive bladder in the rat and investigation of the effects of GABAergic receptor agonists and glutamatergic receptor antagonists on the cystometrogram. J Urol 2005, 173:1805-1811.

33. Vande Griend JP, Linnebur SA: Inhaled anticholinergic agents and acute urinary retention in men with lower urinary tractsymptoms or benign prostatic hyperplasia. Ann Pharmacother 2012, 46:1245-1249.

34. Stephenson A, Seitz D, Bell CM, Gruneir A, Gershon AS, Austin PC, Fu L, Anderson GM, Rochon PA, Gill SS: Inhaled anticholinergic drug therapy and the risk of acute urinary retention in chronic obstructive pulmonary disease: a population-based study. Arch Intern Med 2011, 171:914-920.

35. Mercadante S, Ferrera $P$, Casuccio: Prevalence of opioid-related dysuria in patients with advanced cancer having pain. Am J Hosp Palliat Care 2011, 28:27-30.

36. Kontani $\mathrm{H}$, Kawabata $Y$ : A study of morphine-induced urinary retention in anesthetized rats capable of micturition. Jpn J Pharmacol 1988, 48:31-36.

37. Starr JM: Cholinesterase inhibitor treatment and urinary incontinence in Alzheimer's disease. J Am Geriatr Soc 2007, 55:800-801.

38. Siegler EL, Reidenberg M: Treatment of urinary incontinence with anticholinergics in patients taking cholinesterase inhibitors for dementia. Clin Pharmacol Ther 2004, 75:484-488

39. Hashimoto M, Imamura T, Tanimukai S, Kazui H, Mori E: Urinary incontinence: an unrecognised adverse effect with donepezil. Lancet 2000, 356:568.

40. Brock N, Pohl J, Stekar J: Studies on the urotoxicity of oxazaphosphorine cytostatics and its prevention-I. Experimental studies on the urotoxicity of alkylating compounds. Eur J Cancer 1981, 17:595-607.

41. Golubeva AV, Zhdanov AV, Mallel G, Dinan TG, Cryan JF: The mouse cyclophosphamide model of bladder pain syndrome: tissue characterization, immune profiling, and relationship to metabotropic glutamate receptors. Physiol Rep 2014, 2:e00260. 